\title{
Photon signature analysis using template matching.
}

\begin{abstract}
We describe an approach to detect improvised explosive devices (IEDs) by using a template matching procedure. This approach relies on the signature due to backstreaming $\gamma$ photons from various targets. In this work we have simulated cylindrical targets of aluminum, iron, copper, water and ammonium nitrate (nitrogen-rich fertilizer). We simulate $3.5 \mathrm{MeV}$ source photons distributed on a plane inside a shielded area using Monte Carlo N-Particle (MCNPTM) code version 5 (V5). The $3.5 \mathrm{MeV}$ source gamma rays yield $511 \mathrm{keV}$ peaks due to pair production and scattered gamma rays. In this work, we simulate capture of those photons that backstream, after impinging on the target element, toward a $\mathrm{NaI}$ detector. The captured backstreamed photons are expected to produce a unique spectrum that will become part of a simple signal processing recognition system based on the template matching method. Different elements were simulated using different sets of random numbers in the Monte Carlo simulation. To date, the sum of absolute differences (SAD) method has been used to match the template. In the examples investigated, template matching was found to detect all elements correctly.
\end{abstract}

Keyword: Improvised explosive device; Template matching; Backstreamed photons; Monte Carlo N-Particle (MCNP). 